\title{
Development and Evaluation of Europium-Based Quantitative Lateral Flow Immunoassay for the Chronic Kidney Disease Marker Cystatin-C
}

SATHEESH NATARAJAN ( $\nabla$ satheesh@htic.iitm.ac.in )

Healthcare Technology Innovation Centre https://orcid.org/0000-0002-1045-7472

Ebru saatci

Erciyes Üniversitesi: Erciyes Universitesi

\section{Short Report}

Keywords: Fluorescent quantitative lateral flow assay, Europium nanoparticles, Sandwich assay, Timeresolved fluorescent.

Posted Date: August 10th, 2021

DOI: https://doi.org/10.21203/rs.3.rs-755046/v1

License: (c) (1) This work is licensed under a Creative Commons Attribution 4.0 International License.

Read Full License

Version of Record: A version of this preprint was published at Journal of Fluorescence on January 13th, 2022. See the published version at https://doi.org/10.1007/s10895-021-02886-y. 


\section{Abstract}

This study aimed to establish a Europium label time-resolved fluorescence immunoassay (TRFIA) to detect the chronic kidney disease (CKD) biomarker Cystatin-C. Some Cystatin-c immunoassays are sensitive, accurate, and available for clinical application, but they are expensive and time-consuming procedures. Also, conventional organic dye-based fluorescence lateral flow assay showed more background fluorescence interference and low analytical sensitivity. So this Europium-based sandwich immunoassay was developed to detect the concentration of cystatin-c in a urine sample with captured anti-cystatin-c antibodies immobilized on nitrocellulose membrane and then bonded with detection anticystatin-c labelled with $\mathrm{CM}$-EU, followed by fluorescence measurement using time-resolved fluorometry in 15 minutes. The performance of this TRFIA was evaluated using the clinical urine serum and compared with the ELISA assays. The linear calibration range was $0.015-32 \mu \mathrm{g} / \mathrm{ml}$, and the limit of detection (LOD) quantified was $0.0001 \mu \mathrm{g} / \mathrm{ml}$. This current work has improved the LOD of our previous work from $0.013 \mu \mathrm{g} / \mathrm{ml}$ to $0.001 \mu \mathrm{g} / \mathrm{ml}$. These results indicated that the CM-EU nanoparticle-based LFIA is rapid, more sensitive, reliable, and reproducible for point-of-care testing of Cys-C concentrations in urine

\section{Introduction}

Glomerular filtration rate (GFR) is the most valuable estimate of kidney function and is crucial for the detection and treatment of acute renal failure (ARF) and chronic kidney disease (CKD). Kidney function loss due to decreased estimated glomerular filtration rate (eGFR) is related to short survival. Cystatin-C (Cys-C) is an important biomarker for eGFR determination that is not dependent on muscle mass and relatively constant with age and gender [1]. Cys-C is a $13 \mathrm{kDa}$ protein. It is excreted in only small amounts in urine, filtered by the glomeruli, and absorbed and catabolized by the epithelial cells present in the proximal tubule [2]. The range for Cys-C is $0.67-6.15 \mathrm{mg} / \mathrm{l}$ in urine [3]. Serum levels are $0.80 \pm 0.21$ (range from $0.57-1.12 \mathrm{mg} / \mathrm{dl}$ ) while it is increased up to $2.1+/-1.1 \mathrm{mg} / \mathrm{dl}$ in patients in kidney diseases $[2,4]$. Cys-C is measured in urine using particle-supported turbidimetric or nephelometric immunoassay, photoelectrochemical, fluorescence, near-infrared spectrometry proteomics-based methods, and microfluidics [5-13]. Lateral flow immunoassay is the most rapid test for detecting Cys-C levels, the organic dye-based lateral flow assay. The organic dye Alexafluor-647 often had very high background fluorescence measured due to the membrane scattering. The small stokes shifts of Alexafluor worsen the interferences coming from light scattering and sample matrices. Although the technology has a remarkable performance, it still has some disadvantages, such as low detection sensitivity [14].

The time-resolved fluorescence (TRF) detection method shows the capacity to offer higher than conventional organic dye-based fluorescence because of its low background noise $[15,16]$. Dye-based methods utilize optical filters to dissociate the fluorescence from background light using wavelength differences. The specific fluorescence properties of TRF show high sensitivity, no background fluorescence, significant Stokes shift, dissociation enhancement. This method excites a fluorescent label with a short pulse of light and then waits for a while for the background and other membrane fluorescence to get off a low level and then collects the fluorescent signal. This method eliminates the 
scattered light signals from the blood and can read only the fluorescence from the label. In addition, by removing the autofluorescence from the membrane, time-resolved methods increase the detection sensitivity over conventional fluorescence [17].

The time-resolved fluorescence detection technique is mainly utilized for biological assays using lanthanide chelates Europium (EU) as probes [18] due to its photostability. In addition, carboxylic acid groups on the EU nanoparticle enhance the conjugation efficiency of the protein by increasing the stability of the reporter. To date, EU (III) chelated-dyed nanoparticles are widely used in various immunoassays and lateral flow immunoassays [19]. However, using EU (III) polystyrene chelate microparticles, all quantitative results in LFIA were based solely on the fluorescence signals of the test line. This study used carboxylate-modified polystyrene (CM-EU) microparticles to label Cys-C-LFIA fluorescence in urine samples.

This article reports a CM-EU-based lateral flow immunoassay to obtain an enhanced detection of Cys-C. We have used commercial Europium particles as probes to demonstrate the uniqueness of the technology. The technology should perform several applications, ranging from clinical diagnostics, food and environmental monitoring. Therefore, our strategy offers excellent potential for further development for rapid detection at the treatment site and clinical application.

\section{Experimental Procedures}

Materials

CM-EU was purchased from Thermo Fisher. Tween-20, Triton X-100, N-hydroxy sulfo succinimide sodium salt (Sulpha-NHS), N-(3-dimethyl aminopropyl)-N-ethyl carbodiimide hydrochloride (EDC), 2-(Nmorpholino) ethane sulfonic acid buffer (10 mm MES, pH: 6.1), PBS, and Casein obtained from SigmaAldrich (St. Louis, MO, USA). The anti-Cys-C antibody and Recombinant protein were obtained from HyTest Ltd. (Turku, Finland). Tris-Casein, Abcam, UK). Conjugation pad from Merck (CFSP223000), Glass fiber conjugation pad (GFCP203000), and Glass fiber Diagnostic Pad (GFDX203000) from the Merck Millipore, USA.

Labelling of the Europium with the biomolecules

$2 \mathrm{mg}$ of CM-EU was dissolved in $1 \mathrm{ml}$ of activating buffer (25 mm MES, pH 6.1) consisting of $10 \mathrm{~mm}$ of sulfo-NHS and $1.25 \mathrm{~mm}$ of EDC. Mixtures were shaken at $250 \mathrm{rpm}$ for 60 minutes at room temperature. After that, the solution was centrifuged at $14000 \mathrm{rpm}$ for $25 \mathrm{~min}$ to remove the excess NHS and EDC. The activated CM-EU was washed and re-suspended with $25 \mathrm{~mm}$ Phosphate Buffer (PB). For the conjugation, $50 \mu \mathrm{g} / \mathrm{ml}$ of anti-Cys-C mAb was gently mixed with the activated CM-EU, overnight. The unconjugated antibody was removed by centrifugation at $14000 \mathrm{rpm}$. After washing thrice, $1 \mathrm{ml}$ of blocking buffer (25 $\mathrm{mm} \mathrm{PB}, 2 \%$ casein, $\mathrm{pH} 7.4$ ) was added and incubated for one hour. After incubation, the conjugate was washed thrice. Finally, the conjugates were suspended in $25 \mathrm{~mm}$ PB, 0.5\% BSA, 2.5\% Trehalose, and 10\% sucrose) to the concentration of $10 \mu \mathrm{g} / \mathrm{ml}$ and stored at $4^{\circ} \mathrm{C}$. 
The Europium-based test strip consists of a sample, conjugate, nitrocellulose membrane (NC), and absorbent pad. The sample and conjugate pad were pre-treated with sample pad and conjugate pad treatment buffer ( $1 \mathrm{x}$ Tris-Casein $+0.25 \%$ Tween-20). The conjugates were diluted and mixed in the $1 \mathrm{x}$ TrisCasein to the final concentrations of $0.2,0.3,0.4,0.5 \mathrm{ng} / \mathrm{ml}$. Then the mixture of conjugates was dispensed onto the pre-treated conjugate pad, and the casein with EU was used at $1 \mathrm{mg} / \mathrm{ml}$ and then dried at $37^{\circ} \mathrm{C}$ overnight. Next, anti-Cys-C $(0.5 \mathrm{mg} / \mathrm{ml})$ and anti-mouse $\operatorname{lgG}(1 \mathrm{mg} / \mathrm{ml})$ were dispersed on an NC membrane on the test and control line. After that, the NC membrane was then dried at $37^{\circ} \mathrm{C}$ for two hours and stored at the desiccator. Finally, the whole assembly was cut into a $3.1 \mathrm{~mm}$-wider and placed in cassettes. The strips were stored in a desiccator at room temperature until use.

Fluorescence lateral flow assay procedure

First of all, $80 \mu$ of the different concentrations of standards in the chase buffer $(0.1 \mathrm{~m}$ Tris-Casein + $0.125 \%$ Tween-20) was incubated at room temperature for 15 minutes. Then the mixture is dropped in the sample port after 15 min reaction. Next, the test strip cartridge was inserted and scanned using the IQuant TRF reader. Finally, the $V_{T}$ and $V_{C}$ were measured, and the ratio of $V_{T} / V_{C}$ was calculated. $A$ schematic illustration of the EU (III) chelate nanoparticle-based LFIA procedure is shown in (Fig. 1).

Quantitative data was obtained from the IQuant-TRF [19] Immunoanalyzer (developed by Healthcare Technology Innovation Center, IIT Madras). The instrument scanned the intensity of the test and control line NC to calculate the volume ratio. The device was developed by Karthik et al. 2019 [19].

Accuracy assay

The assay precision was done by analyzing the low, medium, and high concentrations of specimens. In addition, intra- and interassay variations were obtained from 10 independent experiments.

Recovery assay

Recovery was made by adding various concentrations of Cys-C to three clinical urine specimens. The recovery was expressed as a percentage of the amount added. The equation was as follows: Recovery $(\%)=($ measured concentration $/$ enriched concentration $) \times 100$.

Statistical analysis

The anti-Cys-C analytical curve was obtained by plotting the $V_{R} v s$. the concentration of Cys-C. The curves were obtained using Sigmaplot best fitting curve results. Microsoft Excel was utilized to calculate sample means and the standard deviations (SD).

\section{Results}


The developed CM-EU-based LFA to determine anti-Cys-C levels was performed as a sandwich-based lateral flow fluorescent immunoassay, as illustrated in (Fig.1). First, the assay buffer containing Cys-C protein was added to the sample pad. Then, the CM-EU -anti-Cys-C conjugates bind to the Cys-C protein and flow across the NC membrane, which was reacted with the anti-Cys-C capture antibody on the test line, resulting in a fluorescent band on the test, and for the control line, anti-mouse IgG was coated, which binds with the remaining conjugates with the Europium binds to give the fluorescent in the control lines, respectively. Once the reaction is completed, the test strip data was acquired from the IQuant TRF reader by measuring the peak volume of the test line and the control line (Fig.1). As it is a sandwich assay system, anti-Cys-C mAb with CM-EU conjugates form a sandwich with Cys-C in the sample and the antiCys- $C$, coated in the test line. Therefore, the fluorescence intensity at the test line is directly proportional to the concentration of anti-Cys-C in the sample. Next, the $V_{T} / V_{C}$ ratio was used for the measurements and made it more reliable for the analytical sensitivity of clinical applications.

The analytical curve for the LFIA was plotted by a series of different concentrations of anti-Cys-C standards $(0.06,0.12,1,4,8,16$, and $32 \mu \mathrm{g} / \mathrm{ml})$ in sample buffer. A standard curve was obtained after recording the fluorescence intensities plotting the $V_{R}$ against the concentration using the equation: $y=$ $0.0022 x^{2}+0.1928 x+0.0478,(r=0.9952)$ and in (Fig.2). For each concentration, the coefficients of variation (CVs) recorded were less than $10 \%$. The mean of five replicates was recorded using 10 blanks to estimate the LOD. The LODs were recorded from $0.001 \mu \mathrm{g} / \mathrm{ml}$. After establishing the CM-EU-nanoparticle method, a lower LOD was recorded than the Alexa fluor- 647 method $(0.023 \mu \mathrm{g} / \mathrm{ml})$.

\section{Optimization of the conjugation of CM-EU nanoparticle with the anti-Cys-C antibody}

CM-EU is used as the carrier for the conjugation of mAb against Cys-C. Typical EDC and Sulfo-NHS conjugation methods were used. After optimization, $10 \mathrm{~mm}$ of EDC and $1.25 \mathrm{~mm}$ of Sulfo-NHS were used to activate a $2 \mathrm{mg} \mathrm{CM-EU} \mathrm{nanoparticle} \mathrm{solution.} \mathrm{The} \mathrm{mAb} \mathrm{was} \mathrm{then} \mathrm{conjugated} \mathrm{to} \mathrm{the} \mathrm{surface} \mathrm{of} \mathrm{the} \mathrm{CM-}$ EU nanoparticle. After optimization, $50 \mathrm{ng}$ of anti-Cys-C mAb were conjugated to $2 \mathrm{mg}$ of CM-EU nanoparticle, the solution was added to the strip, and the strip observed higher fluorescence intensity. The CM-EU antibody conjugate is diluted to a concentration of $0.2,0.3,0.4,0.5 \mathrm{ng} / \mathrm{ml}$ to optimize the amount of conjugate. Finally, $0.3 \mathrm{ng} / \mathrm{ml}$ was chosen for further experiments shown in (Fig 3).

\section{Optimization of the LFIA Strip}

Before producing the LFIA strip, non-specific protein adsorption was prevented by using an optimized blocking buffer containing $1 x$ Tris-casein, $1.0 \%$ casein, $5 \%$ sucrose, $1.5 \%$ trehalose, and $1.25 \%$ Tween 20 . The casein prevented non-specific protein adsorption, and sucrose and Trehalose aided the conjugate movement in the conjugation pad. In addition, Tween 20 enhanced the specificity of interaction between the mAb and the antigen illustrated in (Fig.4).

\section{Selection of Conjugation pad}


For the experimental purpose, we used a Conjugation pad from a Cellulose conjugation pad from Merck (CFSP223000), a Glass Fiber conjugation pad (GFCP203000), and a Glass fiber Diagnostic Pad (GFDX203000). The proper release of the conjugation material from the above conjugation pad resulted from the Glass fiber Diagnostic Pad (GFDX203000), so we used this pad for all the experiments. Data not shown.

\section{Precision assay}

The intra-assay and inter-assay precision were calculated to show the reproducibility of the developed assay. Three concentrations (low, medium, high) of anti-Cys-C in spiked urine samples were quantified 10 times per day to determine intra-assay precision, and 10 replicates were performed on 3 continuous days the evaluate inter-assay precision. The results are shown in Table.1 The intra-assay CVs were from $4.53 \%$ to $6.89 \%(n=10)$ and inter-assay CVs were from $5.93 \%$ to $8.83 \%(n=30)$. All the obtained CVs were below $10 \%$, which is in the acceptable precision region for the anti-Cys-C quantification.

Table 1. The intra- and inter-assay precision CV\% values.

\begin{tabular}{lllll} 
Samples $(\mu \mathrm{g} / \mathrm{ml})$ & \multicolumn{2}{l}{ Intra-assay precision $(\mathrm{n}=10)$} & \multicolumn{2}{l}{ Intra-assay precision $(\mathrm{n}=10)$} \\
\hline & Mean $\pm \mathrm{SD}(\mu \mathrm{g} / \mathrm{ml})$ & $\mathrm{CV} \%$ & Mean \pm SD $(\mu \mathrm{g} / \mathrm{ml})$ & $\mathrm{CV} \%$ \\
\hline 0.06 & $19.53 \pm 1.29$ & 6.59 & $19.46 \pm 1.64$ & 8.44 \\
\hline 2 & $59.19 \pm 4.32$ & 6.16 & $59.23 \pm 5.87$ & 8.25 \\
\hline 32 & $119.40 \pm 12.3$ & 7.23 & $119.36 \pm 15.37$ & 9.02
\end{tabular}

\section{Recovery Study}

The LFIA recovery percentage of the assay was quantified by dividing the spiked concentration of Cys-C with the observed concentration multiplied by 100 . Control urine (no Cys-C) samples were spiked with four different concentrations of Cys-C standard samples $(0.06,1,16,32 \mu \mathrm{g} / \mathrm{ml})$. The experiment's recovery rates of the four selected samples showed $98,107,100$, and $102 \%$, respectively (Table.2).

Table 2. The Recovery percent of the spikes.

\begin{tabular}{|c|c|c|c|}
\hline Spiked & Concentration $(\mu \mathrm{g} / \mathrm{ml})$ & Observed Concentration $(\mu \mathrm{g} / \mathrm{ml})$ & Recovery (\%) \\
\hline 0.06 & & 0.01465 & 98 \\
\hline 1 & & 1.0645 & 107 \\
\hline 16 & & 16.05 & 100 \\
\hline 32 & & 32.5 & 102 \\
\hline
\end{tabular}




\section{Optimization of the immunoreaction time}

The immunoreaction time of the LFIA is the most significant parameter that can influence the fluorescence intensity development in the Lateral flow strip. For the optimization, the recombinant Cys-C standard sample was used at a 1,10 , and $32 \mu \mathrm{g} / \mathrm{ml}$ concentration. It is also used to test the immunoreaction time of antibody-antigen interaction by evaluating the $V_{R}$ over a range of 3 to 20 minutes incubation囚as illustrated in (Fig.5). Each value was calculated in triplicate, and the scale of error represented the standard deviation of the experiment. The experiment recorded the increase in the $V_{R}$ ratio increased up to $10 \mathrm{~min}$ and then achieved the peak after 15 minutes. These results proved that the $V_{R}$ ratio is considered best to determine the concentration of Cys-C than utilizing the $V_{T}$, and the $V_{R}$ ratio can eliminate the effects of kinetics in the immunoreactions and reduce the turnaround time. Finally, we used 15 minutes as the most appropriate response time for further research.

\section{Sample volume}

To eliminate the nonspecific adsorption of CM-EU conjugates, the amount of sample volume for the assay was optimized. Fluorescence signals with different sample volume were obtained using $100 \mu \mathrm{l}$ (Fig. 6). As shown in Fig. S1, with the increase of sample volume, the $V_{R}$ ratio increases up to $85 \mu$ l but decreases from the $85 \mathrm{ul}$ to $100 \mu \mathrm{l}$ (The results are shown in Figure S1 (Supplementary Materials).

\section{Stability}

The stability study of the CM-EU labelled LFIA was conducted. The strips were preserved in the airtight aluminum foil at $4^{\circ} \mathrm{C}$, and the room temperature was assayed at different time points (months $0,1,2,3$ ). The standard solutions were prepared to contain various concentrations of Cys-C $(1,16,32 \mu \mathrm{g} / \mathrm{ml})$. The relative standard deviation is a relative percentage between the standard deviation and the mean value. It is found that the strip still functions well for detection after 3-month airtight preservation at average temperature. The results are shown in Figure S2 (Supplementary Materials).

\section{Method comparison with standard ELISA}

To evaluate the potential clinical application of our CM-EU-based LF assay, we compared its analytical performance with that of a commercially available ELISA assay kit (Abcam, UK). Seven different concentrations (from 0 to $20 \mu \mathrm{g} / \mathrm{ml}$ ) of Cys-C protein were prepared and tested in both ELISA and the LFIA. As shown in (Fig. 6), the minimum detectable concentration of Cys-C using the commercial assay kit is $0.3 \mu \mathrm{g} / \mathrm{ml}$ (Fig.7b); this is significantly compared with the CM-EU-based lateral flow assay. Furthermore, it is also possible to carry out a highly sensitive quantitative assay of Cys- $\mathrm{C}$ in the lower concentration range. These results mean that the CM-EU labeled LFA method for Cys- $\mathrm{C}$ has a good performance compared with other widely commercialized methods.

\section{Discussion}


Cys-C is a valuable biomarker in GFR measurement as the non-GFR factors do not influence Cys-C. Therefore, a rapid, cost-effective, and quantitative Europium-based Cys-C detection to meet the vast application in the clinical is of urgent need. Therefore, we developed a Europium-based lateral flow kit for the Cys-C determination, as the organic dye has low stoke shift and photobleaching, and we selected Europium as the fluorosphere to overcome the above disadvantages.

A previous study reported developing a user-friendly Alexafluor-647-LF assay to detect Cys-C in the urine samples [14]. The assay was optimized for its sensitivity and allowed detection of Cys-C in the urine sample up to $0.013 \mu \mathrm{g} / \mathrm{ml}$ [14]. This study describes a distinct EU-LFA format for Cys-C to increase the sensitivity. The newly developed kit was a Europium-based sandwich lateral flow assay that uses Europium as the reporter in the LFA kit. The results were obtained within 15 minutes which provided quantitative results from a portable IQuant-TRF reader. The gold-standard method for quantifying the Cys- $C$ is nephelometric and turbidimetric methods, which require larger analytical instruments [5].

On the other hand, the LFA is rapid and easy to use on-site. However, photobleaching, low stroke shift is the issue due to the organic dye reporter used in the development of the kit, which limited its usage in the development of the lateral flow strips [12]. So we selected an alternative to organic dye as Europium to overcome the limitations faced in the organic-based kit development. The strategy for the work is as followed.

First, we selected the correct nitrocellulose membrane through the specifications given by the company [7]. The lateral flow character of the NC membrane is influenced by the chase buffer ingredient, sensitivity, and consistency of the test line, which are mainly considered. We selected HF70, as the CM-EU particle size is $100 \mathrm{~nm}$. Second, the concentration of the antibodies on the lines and the conjugates on the conjugation pad were optimized. Lastly, the volume ratio against the concentration of Cys-C was plotted to get the analytical value. The recovery percent was found between $90-100 \%$ for this assay. The LOD of the aptamer LFIA kit was $0.0001 \mu \mathrm{g} / \mathrm{ml}$. The observed LOD is low compared to our previously developed antibody-based LFA kit, which showed $0.023 \mu \mathrm{g} / \mathrm{ml}$ [14]. The lower LOD can be due to the significant stroke shift and low background noise. The developed kit is highly acceptable for the clinical detection of Cys-C.

\section{Conclusion}

A lateral flow immunoassay based on Cys-C antibodies has been developed, optimized, and successfully validated. The assay is rapid ( $15 \mathrm{~min}$ ), affordable and needs only $80 \mu \mathrm{l}$ of sample. It provides quantitative result which was used to determine the Cys-C concentration in the sample. Under the optimal conditions, the biosensor could be used to detect as low as $1 \mathrm{ng} / \mathrm{ml}$ of Cys-c. The sensitivity was comparative with that of organic dye based fluorescent lateral flow immunoassay. The assay precision is less than $10 \%$ below the LFIA acceptance level as determined by the within-assay and inter-assay $\mathrm{CV}$ with the stability recorded for one year at $4^{\circ} \mathrm{C}$. The stability test experiment showed that by keeping the strips at one week 
at $37^{\circ} \mathrm{C}$, the volume ratio did not change significantly. The Cys-C Rapid Test kit fulfils the demands of clinical practice.

\section{Declarations}

Conflict of interest: The authors declare that the study has not received any funding, and there are no conflicts of interest

Funding No funding was received for this article

Availability of data and material (The data provided is transparent)

Authors' contributions Authors are equally contributed.

Supplementary Materials The following are available online. Figure S1. Optimization sample volume for the reaction. Figure S2. Stability of the fluorescent strip.

\section{References}

1. Helmersson KJ, Lipcsey M, Ärnlöv J, Bell M, Ravn B, Dardashti A, Larsson A (2021) Cystatin C predicts long-term mortality better than creatinine in a nationwide study of intensive care patients. Scientific Reports 11: 5882

2. Stevens LA, Schmid CH, Greene T, Li L, Beck GJ, Joffe MM, Froissart M, Kusek JW, Zhang Y, Coresh J, Levey AS (2009) Factors other than glomerular filtration rate affect serum cystatin C levels. Kidney International 75: 652-660

3. Tian S, Kusano E, Ohara T, Tabei K, Itoh Y, Kawai T, Asano Y (1997) Cystatin C measurement and its practical use in patients with various renal diseases. Clinical Nephrology 48: 104-8

4. Knight EL, Verhave JC, Spiegelman D, Hillege HL, Zeeuw DD, Curhan C, De Jong PE (2004) Factors influencing serum cystatin $\mathrm{C}$ levels other than renal function and the impact on renal function measurement. Kidney International 65: 1416-1421

5. Kyhse-Andersen J, Schmidt C, Nordin G, Andersson B, Nilsson EP, Lindström V, Grubb A (1994) Serum cystatin-C, determined by a rapid, automated particle-enhanced turbidimetric method, is a better marker than serum creatinine for glomerular filtration rate. Clinical Chemistry 40:1921-1926

6. Finney H, Newman DJ, Gruber W, Merle P, Price CP (1997) Initial evaluation of cystatin-C measurement by particle-enhanced immunonephelometry on the Behring nephelometer systems (BNA, BN II). Clinical Chemistry 43: 1016-1022

7. Findlay J, Smith W, Lee J, Nordblom G, Das I, DeSilva B, Khan M, Bowsher R (2002) Validation of immunoassays for bioanalysis: A pharmaceutical industry perspective. Journal of Pharmaceutical and Biomedical Analysis 21: 1249-1273

8. Liu A, Yin K, Mi L, Ma M, Liu Y, Li Y, Wei W, Zhang Y, Liu S (2017) A novel photoelectrochemical immunosensor by integration of nanobody and $\mathrm{ZnO}$ nanorods for sensitive detection of nucleoside 
diphosphatase kinase-A. Analytica Chimica Acta 973: 82-90

9. Lin H, Li L, Lei C, Xu X, Nie Z, Guo M, Huang, Y, Yao S (2013) Immune-independent and label-free fluorescent assay for Cystatin-C detection based on protein-stabilized Au nanoclusters. Biosensors and Bioelectronics 41: 256-261

10. Tao J, Zhao P, Zeng Q (2016) The determination of cystatin-C in serum based on label-free and nearinfrared light emitted PBS BSA QDs. Journal of Materials Chemistry B 4: 4258-4262

11. Hawkridge AM, Muddiman DC (2009) Mass spectrometry-based biomarker discovery: Toward a global proteome index of individuality. Annual Review of Analytical Chemistry 2: 265-277

12. Wang T, Zhang M, Dreher DD, Zeng Y (2013) Ultrasensitive microfluidic solid-phase ELISA using an actuatable microwell-patterned PDMS chip. Lab A Chip 13: 4190-4197

13. Ouyang W, Han J (2019) Universal amplification-free molecular diagnostics by billion-fold hierarchical nanofluidic concentration. Proceedings of the National Academy of Sciences USA 116: 16240-16249

14. Natarajan S, DeRosa MC, Shah MI, Jayaraj J (2021) Development and Evaluation of a Quantitative Fluorescent Lateral Flow Immunoassay for Cystatin-C, a Renal Dysfunction Biomarker. Sensors 21: 3178

15. Von LP, Rosenberg J, Pettersson K, Takalo H (2003) A europium chelate for quantitative point-of-care immunoassays using direct surface measurement. Analytical Chemistry 75: 3193-3201.

16. Song X, Knotts M (2008) Time-resolved luminescent lateral flow assay technology. Analytica chimica acta. 26: 186-192

17. Harma H, Soukka T, Lovgren T (2001) Europium nanoparticles and time-resolved fluorescence for ultrasensitive detection of prostate-specific antigen. Clinical Chemistry 247: 561-568

18. Song X, Huang, Wu B (2008) Bright and monodispersed phosphorescent particles and their applications for biological assays. Analytical Chemistry 80: 5501-5507

19. Karthik S, Shah MI, Natarajan S, Shetty MJ, Joseph JA (2019) Motion Free Image-Based TRF Reader for Quantitative Immunoassay. In 2019 IEEE Healthcare Innovations and Point of Care Technologies, (HI-POCT) 20: 163-166

\section{Figures}




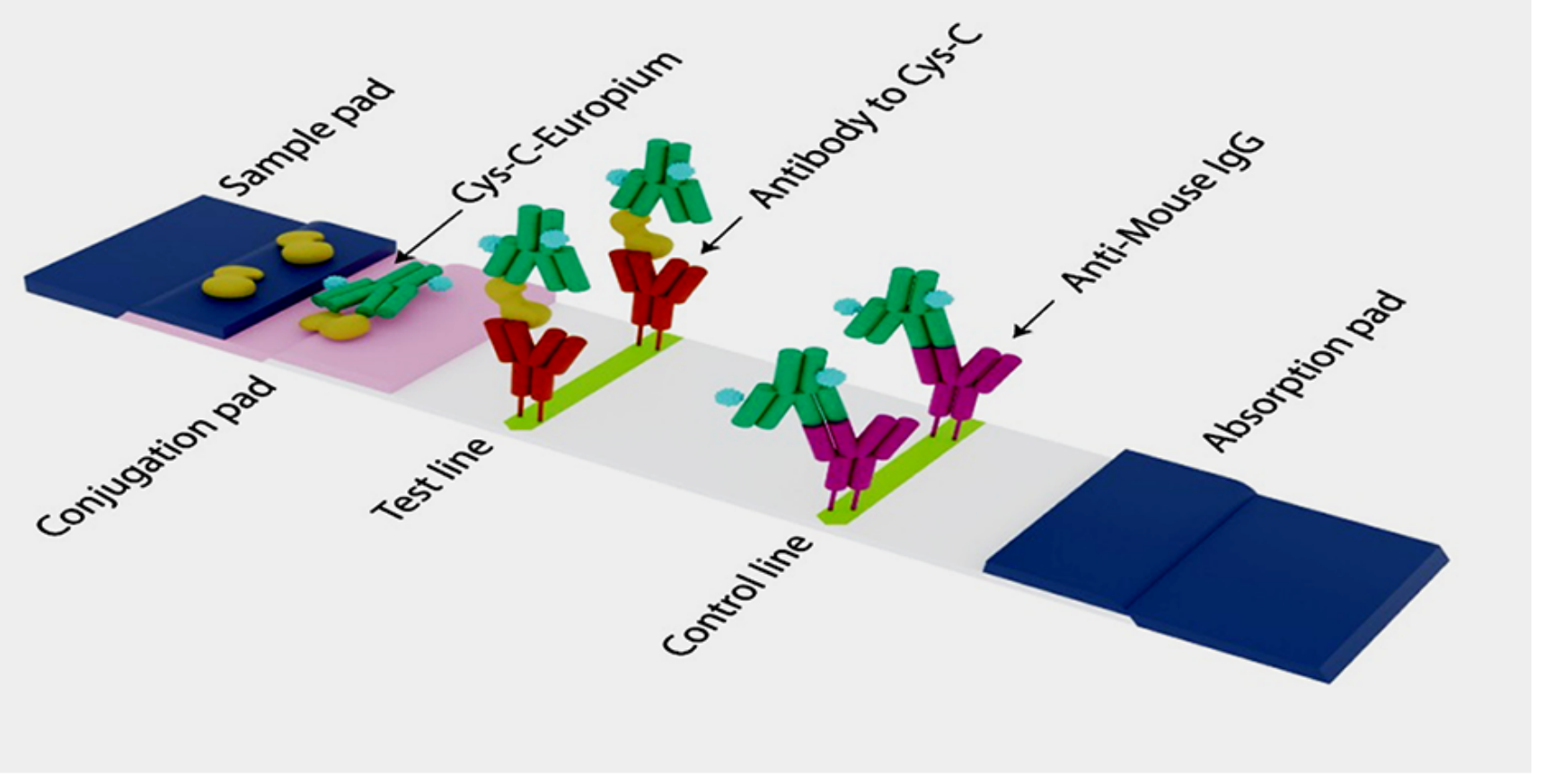

\section{A}
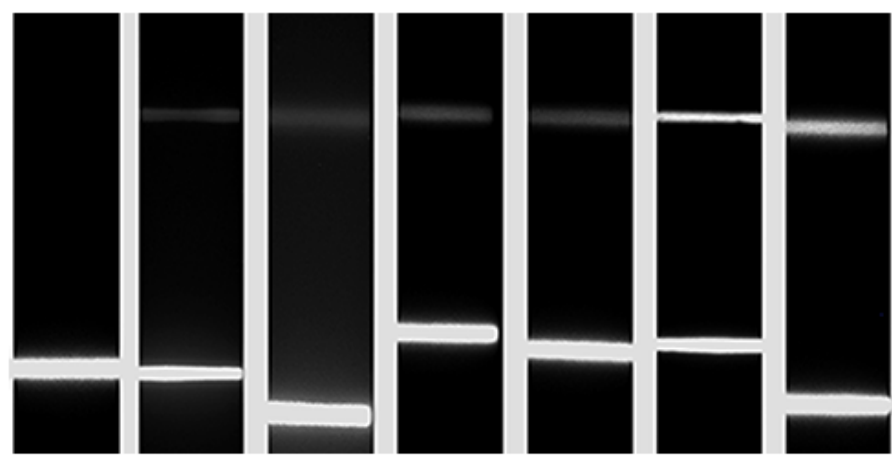

B

Figure 1

(a) Schematic representation of the CM-EUs-based lateral flow assay with test strip components and assemblies. (b) The LFA strip photograph for the $0.06,0.12,1,4,8,16$, and $32 \mu \mathrm{g} / \mathrm{ml}$ of Cys-C. 


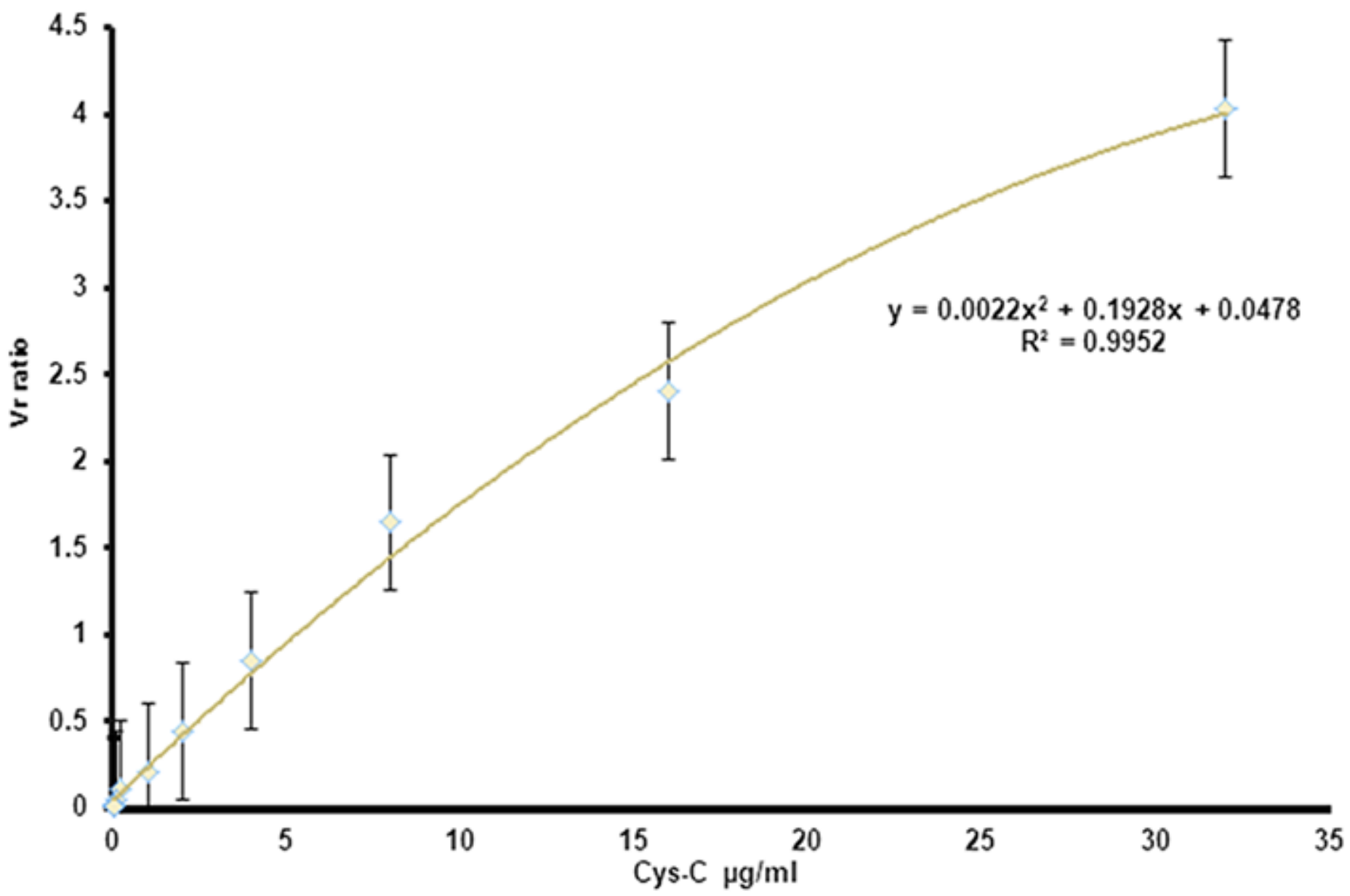

Figure 2

The standard curve of CM-EU-based LFIA strips for anti-Cys-C is obtained from standards from 0.015 to $32 \mu \mathrm{g} / \mathrm{ml}$ by determining fluorescence peak heights on the readout curve. 


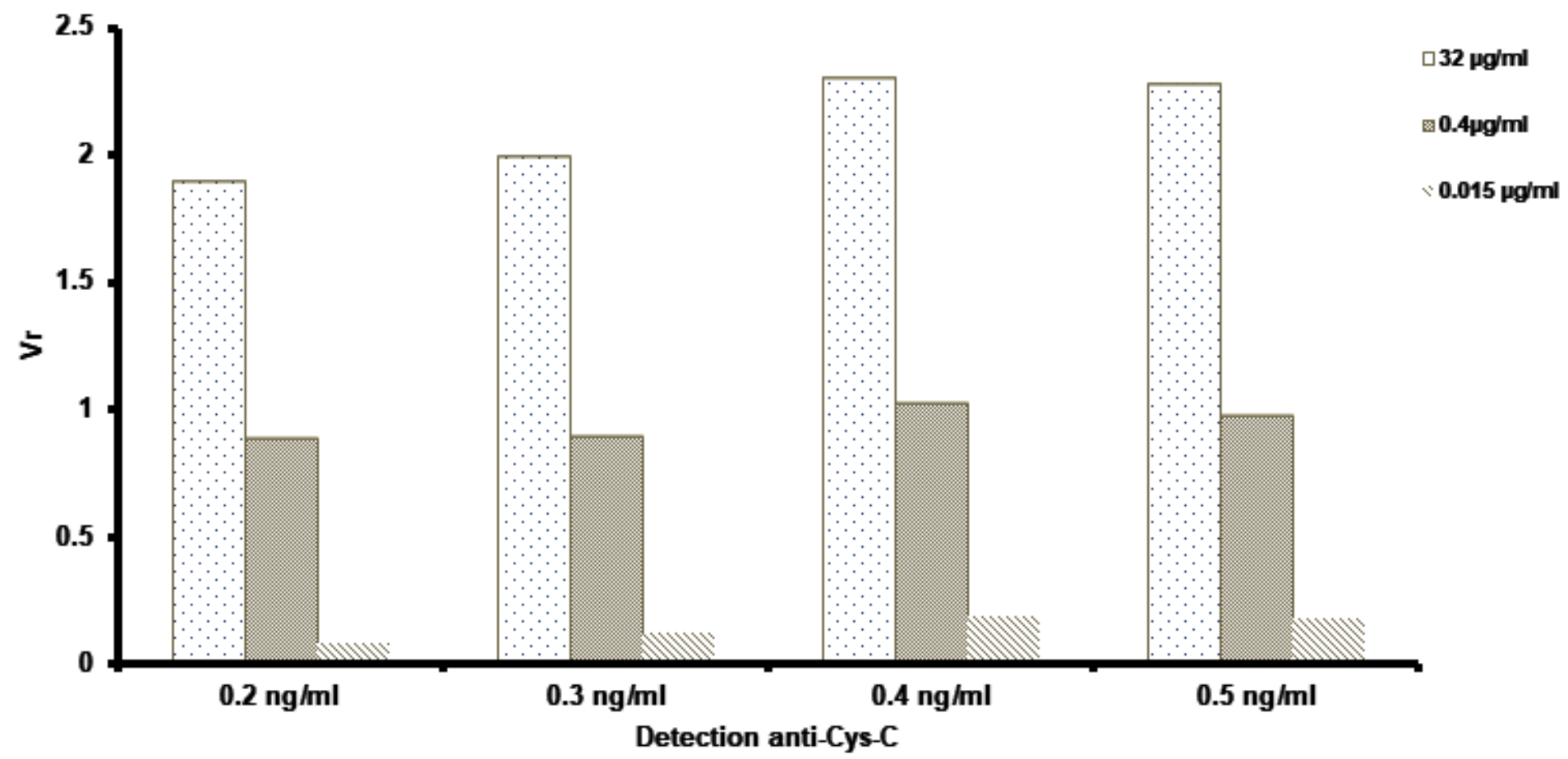

Figure 3

The concentration of the Dab in the conjugation pad for the non-specific binding 


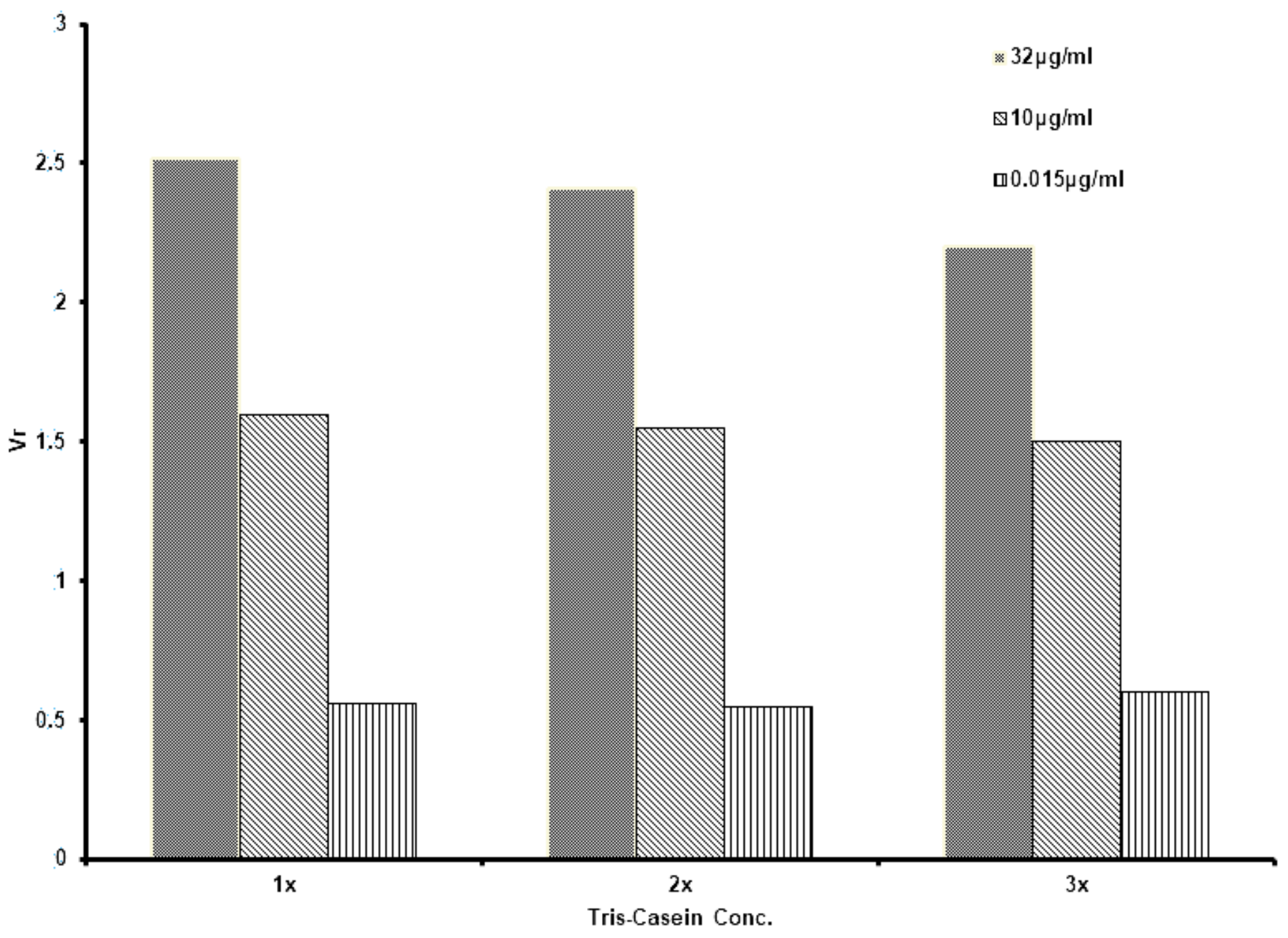

Figure 4

The concentration of the Tris-Casein for the non-specific binding 


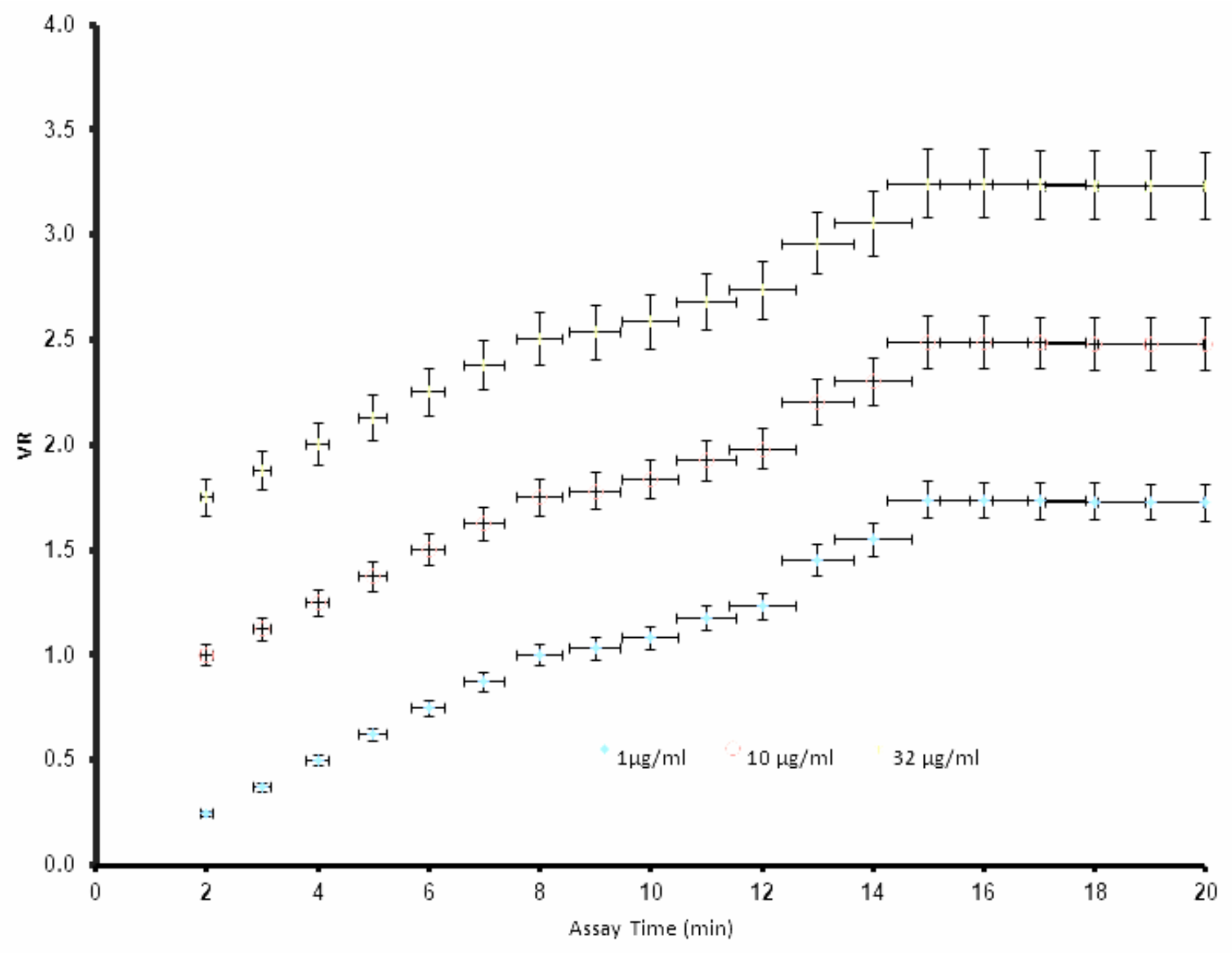

Figure 5

The immunoreaction time of the CM-EU based lateral flow assay and the corresponding VR (BG-Rem) values 

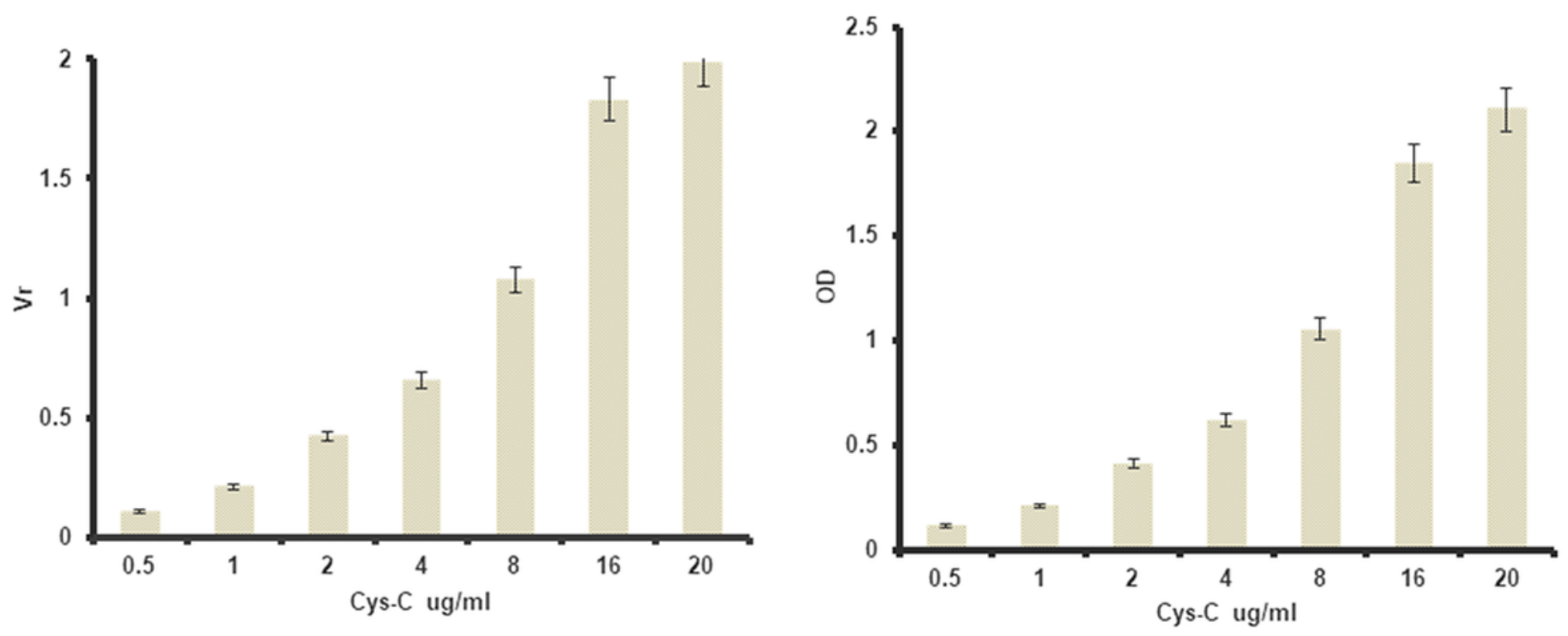

Figure 6

Comparison of the assay results for different concentrations of Cystatin-c using (CM-EU-based lateral flow assay method and (b) a commercially available ELISA method. The error bars indicate the standard deviations calculated from five independent measurements

\section{Supplementary Files}

This is a list of supplementary files associated with this preprint. Click to download.

- Supplementarymaterials.docx 